\title{
Three Synchronous Bilateral Solid Papillary Carcinomas of the Breast with Different Radiologic Findings: A Case Report
}

\author{
Ji Hae Moon, ${ }^{1}$ Shin Young Kim, ${ }^{1, *}$ Sun Huh, ${ }^{1}$ Hyun Ju Lee, ${ }^{2}$ and Jong Eun Lee ${ }^{3}$ \\ ${ }^{1}$ Department of Radiology, Cheonan Hospital, Soonchunhyang University College of Medicine, Cheonan, Korea \\ ${ }^{2}$ Department of Pathology, Cheonan Hospital, Soonchunhyang University College of Medicine, Cheonan, Korea \\ ${ }^{3}$ Department of Surgery, Cheonan Hospital, Soonchunhyang University College of Medicine, Cheonan, Korea \\ "Corresponding author: Shin Young Kim, Department of Radiology, Cheonan Hospital, Soonchunhyang University College of Medicine, 31 Soonchunhyang 6-gil, Dongnam-gu, \\ Chungcheongnam-do, Cheonan, Korea. Tel: +82-415703515, Fax: +82-415799026, E-mail: c87093@schmc.ac.kr
}

Received 2017 July 29; Revised 2018 February 13; Accepted 2018 February 18.

\begin{abstract}
Papillary carcinoma of the breast is a rare malignant tumor that is known to account for only approximately $1 \%$ - $2 \%$ of breast carcinomas in women. Among papillary carcinomas, solid papillary carcinoma (SPC) is a rare breast carcinoma that occurs primarily in elderly women. It is known that approximately $95 \%$ of the cases occur in a unilateral breast. The imaging findings for SPCs are not well established, and most of the published articles are about pathological findings. Moreover, there have been no reports of SPCs occurring in both breasts at the same time. We report here a case of three synchronous bilateral breast SPCs in patient with palpable mass, each showing different radiologic findings. Although imaging findings that distinguish between malignant and benign papillary lesions are unclear, familiarity with the features of SPCs may be helpful in establishing imaging findings for these lesions.
\end{abstract}

Keywords: Mammography, Ultrasonography, Magnetic Resonance Imaging, Breast, Breast Neoplasms

\section{Introduction}

Papillary carcinoma of the breast is known to account for approximately $1 \%$ - $2 \%$ of breast carcinomas in women (1). Papillary lesions are categorized as intraductal papilloma, intraductal papillary carcinoma, encapsulated papillary carcinoma, and solid papillary carcinoma (SPC) (2). SPC is a tumor composed of circumscribed nodules of epithelial cells that are typically ovoid or spindle-shaped with low nuclear grade, and it develops predominantly in elderly patients (3). There are few case reports on SPCs and most studies report on pathologic findings in SPC patients $(4,5)$. SPCs are mostly located in the central part of the breast, and 95\% of SPCs are present in unilateral breasts (6). To our knowledge, there have been no reported cases of SPCs in both breasts of one patient. We here report a case of synchronous SPCs in both breasts in a single patient, which may be helpful in becoming familiar with the varying imaging findings of SPC.

\section{Case Presentation}

A 69-year-old postmenopausal woman presented with a palpable mass in the upper outer quadrant of the left breast. She had no children and no family history of breast or ovarian carcinoma.

Mammography showed round shaped hyperdense masses with indistinct margins in the left upper outer quadrant (Figure 1). We identified this mass as breast imaging reporting and data system (BI-RADS) category 0 and recommended breast ultrasonography (US).

US revealed an irregularly shaped, indistinct, hypoechoic mass in the left 3 o'clock position that measured approximately $1.2 \mathrm{~cm}$ at the palpable site (Figure 2A) and another irregular, spiculated, hypoechoic mass in the left 2 o'clock position that measured $0.9 \mathrm{~cm}$ (Figure 2B). USguided core biopsy was performed on these masses, and we noted a left axillary lymph node with a suspected metastasis. We gave the diagnosis of high suspicion for malignancy with BI-RADS 4c classification. Histopathological examination reported both masses as solid papillary carcinomas (SPCs) with histological grade I/III.

On magnetic resonance imaging (MRI) for preoperation evaluation, there were irregularly shaped, homogeneous enhancing masses approximately $7 \mathrm{~cm}$ in size with spiculated margins across the left upper outer quadrant and lower outer quadrant (Figure $3 \mathrm{~A}$ and $B)$. These masses showed early enhancement and rapid 


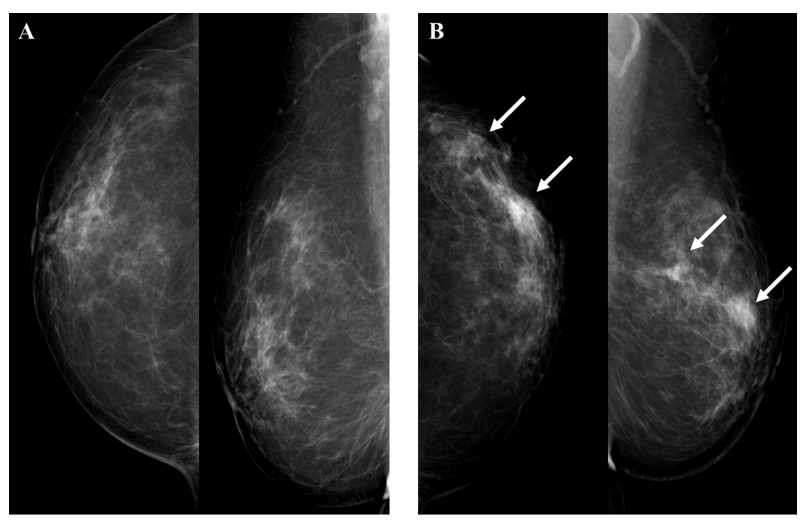

Figure 1. A 69-year-old woman with a palpable mass in the upper outer quadrant of the left breast. A, Mammography of the right breast shows no remarkable finding. B, Mammography of the left breast shows round shaped, indistinct marginated hyperdense masses in the left upper outer quadrant (arrows).
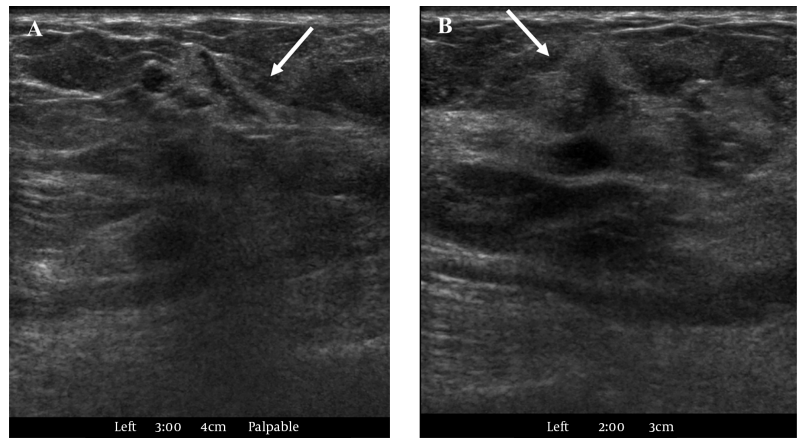

$\overline{\text { Figure 2. Ultrasonography of left breast carcinoma. A, An irregularly shaped, indis- }}$ tinct, hypoechoic mass in the left 3 o'clock position measuring $1.2 \mathrm{~cm}$ (arrow); B, An irregular, spiculated, hypoechoic mass in the left 2 o'clock position measuring 0.9 $\mathrm{cm}$ (arrow).

washout (Figure 3C). Although the right breast showed no abnormality on preoperative US, MRI revealed a lobular, homogeneous enhancing mass approximately $0.8 \mathrm{~cm}$ in size with smooth margins in the subareolar area and a round, central enhancing mass of approximately $0.8 \mathrm{~cm}$ in size with irregular margins in the right upper center (Figure 3D and E). These masses showed early enhancement but no washout in reverse subtraction image (Figure $3 F)$. On chest computed tomography (CT), there was an irregularly shaped, heterogeneously enhancing mass in the left breast and a triangular enhancing mass in the subareolar area and a subtle enhancing mass in the upper center of the right breast (Figure 4).

Second-look US was performed on the right breast, and it revealed a circumscribed, oval, hypoechoic mass in the right 12 o'clock position that measured approximately 0.7 $\mathrm{cm}$ and an irregularly shaped hypoechoic mass with in- distinct margins in the right 9 o'clock position that measured $0.6 \mathrm{~cm}$ (Figure 5). We assumed that the lesion in the right breast at 12 o'clock would be a papilloma, and diagnosed it as low suspicion for malignancy with BI-RADS 4a classification. We also assumed that the lesion on the right breast at 9 o'clock would be a papilloma or highrisk breast lesion, and diagnosed it as moderate suspicion for malignancy with BI-RADS $4 \mathrm{~b}$ classification. The patient underwent a left simple mastectomy with sentinel lymph node biopsy, axillary lymph node dissection and a right needle localized excisional biopsy. Histopathological examination revealed three SPCs in both breasts (Figures 6,7$)$. We determined the left breast mass as an SPC with invasion, and the invasive component was $6.6 \times 3.5$ $\times 2.5 \mathrm{~cm}$ in size. We considered the right breast masses as SPCs with invasion at 12 o'clock position and an SPC in situ at 9 o'clock position. Immunohistochemical stain showed estrogen receptor and progesterone receptor positivity and human epidermal growth factor receptor 2 negativity. In the frozen biopsy of the sentinel lymph node, the macrometastasis was identified. In the final pathologic report, two of the 30 axillary lymph nodes surgically removed were confirmed as metastatic lymph nodes and diagnosed as pN1a. The patient underwent additional doxorubicin/cyclophosphamide chemotherapy and radiation therapy, and is currently being followed up without recurrence for nine months.

\section{Discussion}

According to the 4th edition of world health organization (WHO) classification of tumors of the breast, papillary lesions are classified as intraductal papilloma, intraductal papillary carcinoma, encapsulated papillary carcinoma, and SPC. In case of SPC, it is subdivided into SPC in situ and invasive SPC. The diagnostic criteria for SPC is small and monotonous cells with hyperchromatic nuclei that may show neuroendocrine or mucinous differentiation, and classified as in situ when surrounded by a myoepithelial layer (2). SPC is a rare breast carcinoma that occurs primarily in elderly women, and it is known that approximately $95 \%$ occur in unilateral breasts (6). In our case, it is noteworthy that unlike with most SPCs, we found masses in both breasts at the same time, and three pathologically confirmed SPCs showed different radiographic findings. There has been little research on synchronous bilateral SPCs. Ultrasound with color Doppler is known to be the most sensitive modality for evaluating papillary breast lesions (6). Papillary carcinomas appear as a pattern of single or multiple, circumscribed, solid or complex mixed cystic and solid masses on ultrasonography. Because papillary 

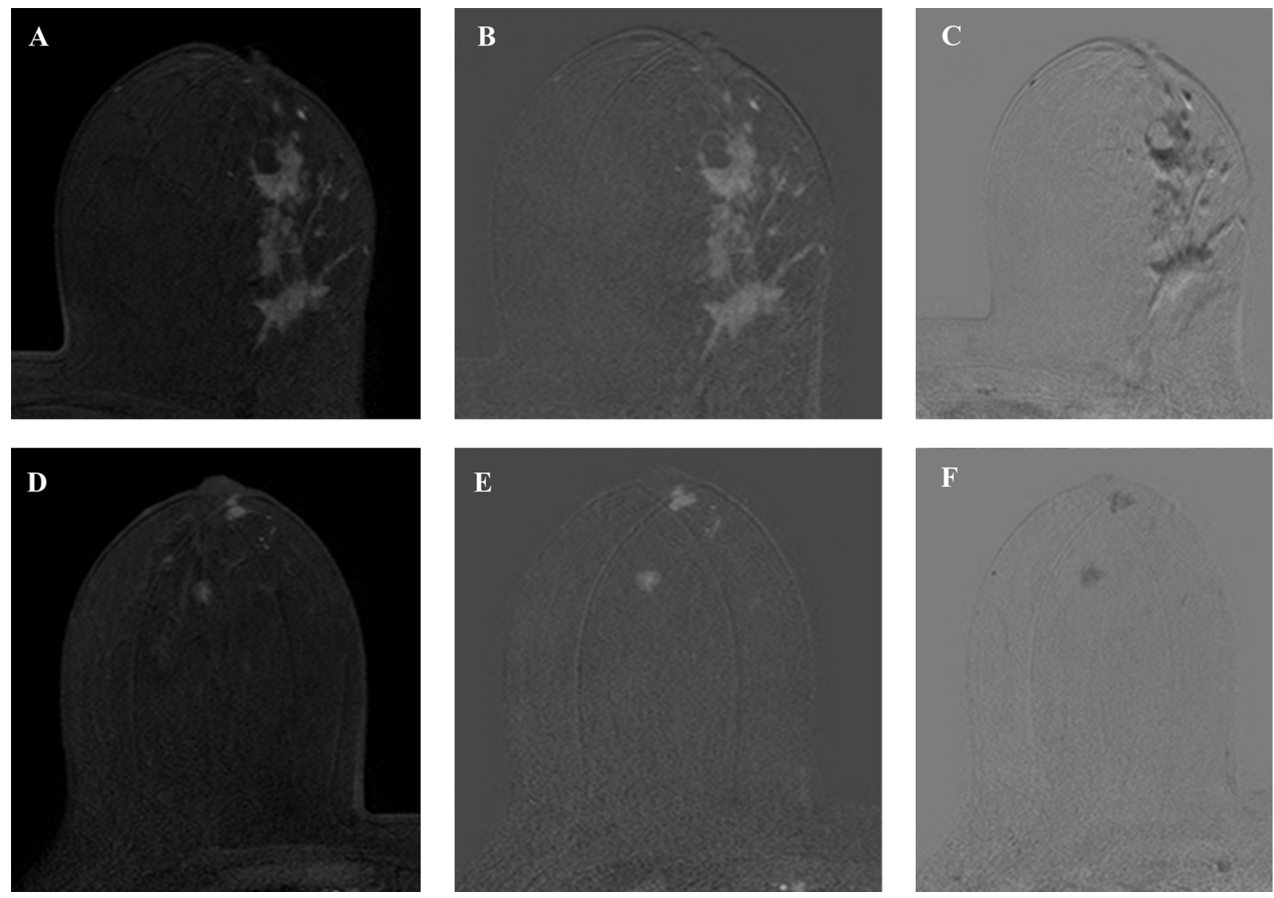

Figure 3. MRI was performed using the 3.0-T system. A, An axial early (2 min) contrast-enhanced T1-weighted image after bolus administration of gadolinium-based contrast shows $7 \mathrm{~cm}$ in size, irregularly shaped, homogeneous enhancing masses with spiculated margins across the left upper outer quadrant and lower outer quadrant. B, An axial subtraction image of the left breast shows the masses with early enhancement. C, These masses show rapid washout in the reverse subtraction image. D - F, Images of the right breast in the same sequence as the left breast show a $0.8 \mathrm{~cm}$, lobular, homogeneous enhancing mass with smooth margins in the subareolar area and a $0.8 \mathrm{~cm}$, round, central enhancing mass with irregular margins in the right upper center. These masses show early enhancement but no washout in the reverse subtraction image.
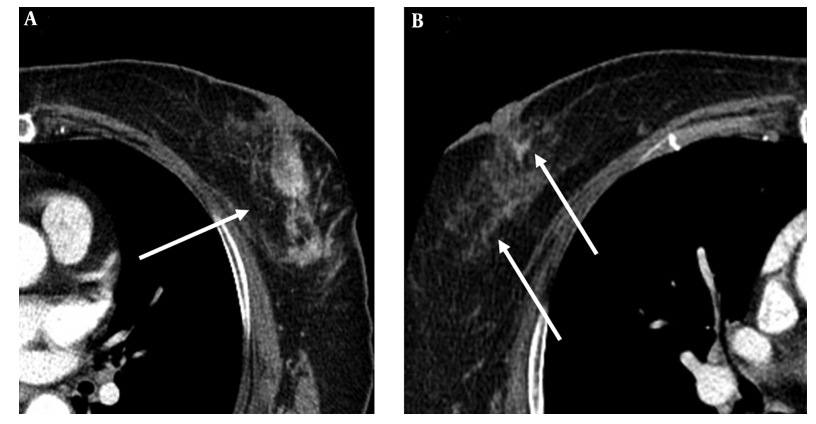

Figure 4. Contrast-enhanced chest CT. A, An irregularly shaped, heterogeneously enhancing mass in the left breast (arrow); B, A triangular enhancing mass in the subareolar area and subtle enhancing mass in upper center of the right breast (arrows).

carcinomas tend to bleed centrally, color Doppler sonography may be helpful in diagnosis (7). In our case, we saw an irregular shaped hypoechoic mass with indistinct margin and an irregular shaped hypoechoic mass with spiculated margin in the left breast and an oval hypoechoic mass with a circumscribed margin and an irregular shaped hypoechoic mass with indistinct margin in the right breast. The two masses we saw on US of the left breast appeared as
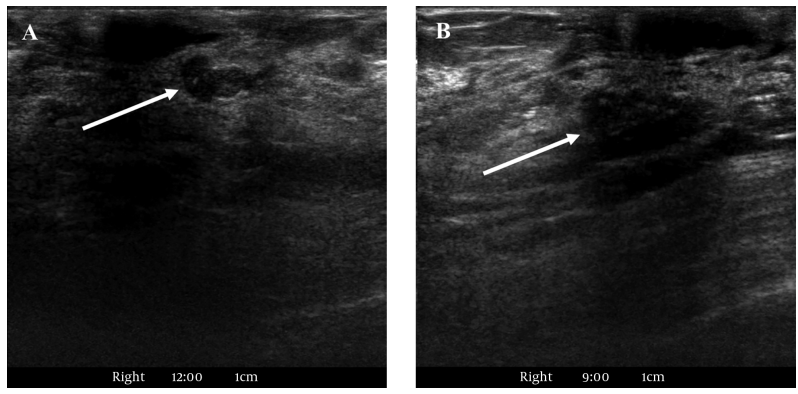

Figure 5. Second-look US of the right breast. A, A circumscribed, oval, hypoechoic mass in the right 12 o'clock position measuring $0.7 \mathrm{~cm}$; B, An irregularly shaped hypoechoic mass with indistinct margins in the right 9 o'clock position measuring 0.6 $\mathrm{cm}$.

one large connected mass on subsequent MRI and CT.

On mammography, papillary carcinomas can be seen as solitary round, oval, or lobulated circumscribed masses or as clusters of well-defined masses with or without associated microcalcifications (7). In our case, we detected round hyperdense masses with indistinct margins.

On MRI, papillary carcinomas may appear as irregular enhancing nodules or enhancing complex cysts. Because 

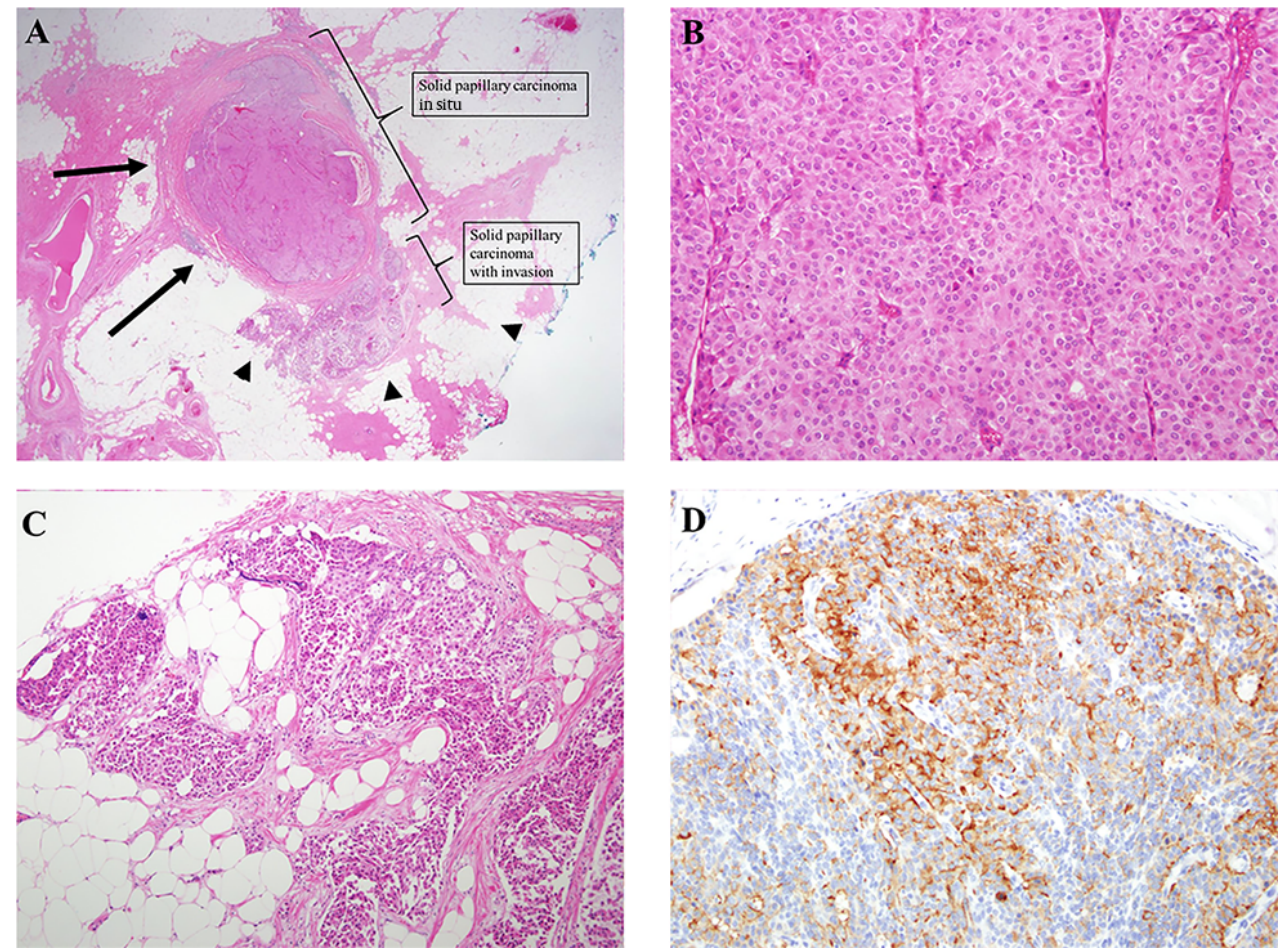

Figure 6. Solid papillary carcinoma (SPC) with invasion in the right breast at 12 o'clock position. A, Circumscribed, large cellular nodules are found $(\mathrm{H} \& \mathrm{E}$ staining $\times 1.25)$. The size of the SPC in situ is $0.9 \times 0.8 \times 0.5 \mathrm{~cm}$ and the size of the invasive SPC located immediately adjacent to the SPC in situ is $0.5 \times 0.4 \times 0.4 \mathrm{~cm}$. Therefore, about $70 \%$ of the total tumors are SPC in situ component with pushing boundaries (arrows), the other $30 \%$ is invasive SPC with infiltrative border (arrowheads). B, The cellular proliferation is homogeneous and cohesive, lacking obvious papillary architecture. The cell shape is polygonal. Cytoplasm is moderate in amount and finely granular eosinophilic ( $\mathrm{H} \& \mathrm{E}$ staining $\times 20)$. C, The cellular nests appear invasive because of their presence of a geographic jigsaw pattern with more ragged and irregular margins $(\mathrm{H} \& \mathrm{E}$ staining $\times 10)$ $\mathrm{D}$, Immunohistochemical evidence of neuroendocrine differentiation, consisting of staining for synaptophysin, is observed $(\times 20)$

the morphologic features of papillary carcinomas on MRI and their kinetic curves are variable and may overlap with those of benign papillomas, information on the characteristic imaging findings for papillary carcinoma is known to be limited in MRI (8). In our case, we saw large, irregularly shaped, homogeneous enhancing masses with spiculated margins, a homogeneous enhancing mass with a smooth margin, and a round, central enhancing mass with an irregular margin. The masses on the left breast showed rapid washout in the reverse subtraction image, but the masses on the right breast showed no washout.

Pathological diagnosis of left and right breast masses were the same solid papillary carcinoma with invasion, but there were two major pathologic differences, the first was the tumor border and the second was the position and ratio of the invasive and in situ components. We assume that those may have caused the difference in radiological findings. In the left breast, the boundary of the entire tumor of $6.6 \times 3.5 \times 2.5 \mathrm{~cm}$ had an infiltrative border. SPC in situ component is about $60 \%$ of the total left breast tumors, which lie between intratumoral and peritumoral.
The mass from the right 12 o'clock position, the size of the SPC in situ is $0.9 \times 0.8 \times 0.5 \mathrm{~cm}$ and the size of the invasive SPC located immediately adjacent to the SPC in situ is $0.5 \times 0.4 \times 0.4 \mathrm{~cm}$. Therefore, about $70 \%$ of the total tumors are SPC in situ component with pushing boundaries. The other 30\% are invasive SPCs with infiltrative borders. In the 9 o'clock position of the right breast, the whole tumor is only SPC in situ component $2.0 \times 1.0 \times 1.0 \mathrm{~cm}$ in size with pushing boundaries.

Because SPC is a rare breast cancer, the kinetics of SPC is not well known. In this case, it can be considered that the kinetics are different due to the difference of cellular stage. However, since benign papilloma can also show a rapid washout pattern (8), and the SPCs were reported to exhibit a plateau pattern showing persistent enhancement, it is not appropriate to determine malignancy only by the enhancement pattern (9).

Although papillary carcinomas are difficult to differentiate from benign papillomas using imaging features alone, we categorized the lesion in the right breast as BIRADS 4a because of our low suspicion for malignancy, but 

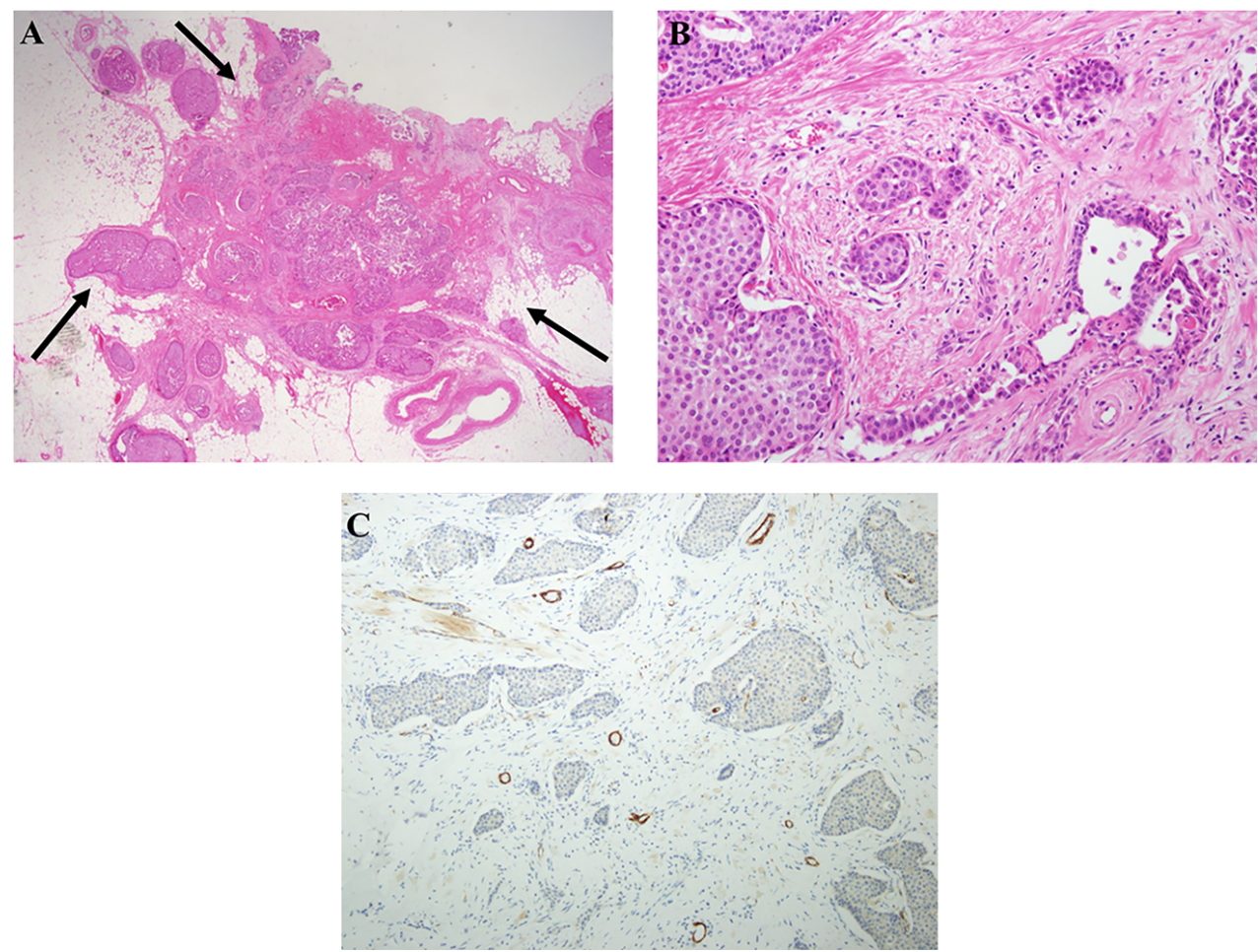

Figure 7. Solid papillary carcinoma (SPC) with invasion in the left breast. A, Scanning magnification of breast tissue showing multiple circumscribed cellular masses comprised of closely apposed, expanded and solidified round structures arranged in contiguous, embedded in dense fibrous stroma (H \& E staining $\times 1.25$ ). The boundaries of the entire tumor of $6.6 \times 3.5 \times 2.5 \mathrm{~cm}$ are infiltrative border (arrows). SPC in situ component is about $60 \%$ of the total left breast tumors, which lie between intratumoral and peritumoral. B, Obvious invasive growth of the conventional infiltrative type are found (H \& E staining $\times 20)$. C, Immunohistochemistry for smooth-muscle myosin heavy chain (Smm-hc) shows complete absence of a peripheral myoepithelial lining around the cellular nodules $(\mathrm{H} \& \mathrm{E}$ staining $\times 10)$.

it is also notable that SPC was confirmed after surgery.

Among the papillary carcinomas, SPC is rare, and a study by Guo et al. reported excellent prognoses for SPCs compared with conventional breast carcinoma; thus, it needs to be distinguished in order to avoid overtreatment (10). The SPC imaging findings are not well established. We report a case of three synchronous bilateral breast SPCs in the same patient at the same time with different ultrasound findings, which could help practitioners familiarize themselves with different imaging findings for SPCs seen under the same conditions.

\section{Footnotes}

Authors' Contributions: None declared.

Conflicts of Interest: None declared.

Financial Disclosure: None declared.

Funding/Support: This work was supported by the Soonchunhyang University research fund.

\section{References}

1. Soo MS, Williford ME, Walsh R, Bentley RC, Kornguth PJ. Papillary carcinoma of the breast: imaging findings. AJR Am J Roentgenol. 1995;164(2):321-6. doi: 10.2214/ajr.164.2.7839962. [PubMed: 7839962].

2. Sinn HP, Kreipe H. A Brief Overview of the WHO Classification of Breast Tumors, 4th Edition, Focusing on Issues and Updates from the 3rd Edition. Breast Care (Basel). 2013;8(2):149-54. doi: 10.1159/000350774. [PubMed: 24415964]. [PubMed Central: PMC3683948].

3. Mulligan AM, O'Malley FP. Papillary lesions of the breast: a review. Adv Anat Pathol. 2007;14(2):108-19. doi: 10.1097/PAP.ob013e318032508d. [PubMed: 17471117].

4. J BL, Kini RG, Amber S. Invasive (solid) Papillary Carcinoma of the Breast: A Report of Two Cases. J Clin Diagn Res. 2013;7(6):1150-1. doi: 10.7860/JCDR/2013/5134.3045. [PubMed: 23905125]. [PubMed Central: PMC3708220].

5. Otsuki Y, Yamada M, Shimizu S, Suwa K, Yoshida M, Tanioka F, et al. Solid-papillary carcinoma of the breast: clinicopathological study of 20 cases. Pathol Int. 2007;57(7):421-9. doi: 10.1111/j.14401827.2007.02118.x. [PubMed: 17587241].

6. Saremian J, Rosa M. Solid papillary carcinoma of the breast: a pathologically and clinically distinct breast tumor. Arch Pathol Lab Med. 2012;136(10):1308-11. doi: 10.5858/arpa.2011-0227-RS. [PubMed: 23020734].

7. Muttarak M, Lerttumnongtum $P$, Chaiwun B, Peh WC. Spectrum of papillary lesions of the breast: clinical, imaging, and pathologic correlation. AJR Am J Roentgenol. 2008;191(3):700-7. doi: 10.2214/AJR.07.3483. [PubMed: 18716096]. 
8. Eiada R, Chong J, Kulkarni S, Goldberg F, Muradali D. Papillary lesions of the breast: MRI, ultrasound, and mammographic appearances. $A J R$ Am J Roentgenol. 2012;198(2):264-71. doi: 10.2214/AJR.11.7922. [PubMed: 22268167].

9. Zhang L, Zhuang L, Shi C, Miao Y, Zhang W, Song Q, et al. A pilot evaluation of magnetic resonance imaging characteristics seen with solid papillary carcinomas of the breast in 4 patients. BMC Can- cer. 2017;17(1):525. doi: 10.1186/s12885-017-3518-8. [PubMed: 28784112] [PubMed Central: PMC5547522].

10. Guo S, Wang Y, Rohr J, Fan C, Li Q, Li X, et al. Solid papillary carcinoma of the breast: A special entity needs to be distinguished from conventional invasive carcinoma avoiding over-treatment. Breast 2016;26:67-72. doi:10.1016/j.breast.2015.12.015. [PubMed: 27017244]. 